\title{
A Cascade of Boosted Generative and Discriminative Classifiers for Vehicle Detection
}

\author{
Pablo Negri, Xavier Clady, Shehzad Muhammad Hanif, and Lionel Prevost \\ Institut des Systèmes Intelligents et de Robotique, CNRS FRE 2507, Université Pierre et Marie Curie-Paris 6, \\ 3 Rue Galilée, 94200 Ivry sur Seine, France \\ Correspondence should be addressed to Lionel Prevost, lionel.prevost@upmc.fr
}

Received 1 October 2007; Revised 4 January 2008; Accepted 16 January 2008

Recommended by Hubert Cardot

\begin{abstract}
We present an algorithm for the on-board vision vehicle detection problem using a cascade of boosted classifiers. Three families of features are compared: the rectangular filters (Haar-like features), the histograms of oriented gradient (HoG), and their combination (a concatenation of the two preceding features). A comparative study of the results of the generative (HoG features), discriminative (Haar-like features) detectors, and of their fusion is presented. These results show that the fusion combines the advantages of the other two detectors: generative classifiers eliminate "easily" negative examples in the early layers of the cascade, while in the later layers, the discriminative classifiers generate a fine decision boundary removing the negative examples near the vehicle model. The best algorithm achieves good performances on a test set containing some 500 vehicle images: the detection rate is about $94 \%$ and the false-alarm rate per image is 0.0003 .
\end{abstract}

Copyright (C) 2008 Pablo Negri et al. This is an open access article distributed under the Creative Commons Attribution License, which permits unrestricted use, distribution, and reproduction in any medium, provided the original work is properly cited.

\section{INTRODUCTION}

The increasing number of cars has increased the demand of driver assistance systems which makes driving more comfortable and safe [1]. Many researches have been conducted by the intelligent transport systems (ITSs) community in this field. It deals with the installation of high-tech devices and other controllers on vehicles and road networks. Among the systems to be integrated on intelligent vehicles, it is necessary to distinguish those related to perception. They can be either proprioceptive (to deal with the vehicle internal state) or exteroceptive (to deal with the vehicle external environment).

In this framework, many vision-based sensors are being studied now. Indeed, an on-board vision system can provide information about the localization and the size of other vehicles in the environment, the road, the traffic signs, and the other users of the road network.

This article deals with a monocular vision-based system. We present an algorithm for on-board vehicle detection. We have used a cascade of boosted classifiers quite similar to the one proposed by Viola and Jones in 2001 [2] for face detection. Here, two types of features are employed and hence compared: the rectangular filters (Haar-like features) and the histograms of oriented gradient (HoG). These two features are frequently used in the domain of object detection and recognition.

The originality of our work is (1) the use of generative classifiers in association with HoG features, (2) the comparative study of discriminative classifiers (based on Haarlike features) and generative (HoG) classifiers, and (3) the concatenation of both features.

The paper is divided as follows. The following section presents previous researches in vehicle detection with a particular focus on boosted detector and most commonly used feature set. Section 3 describes precisely the feature spaces we studied. Weak learners used in the boosting process are described in Section 4. Section 5 details several detection architectures: respectively, a single stage detector and two cascade (multistages) detectors. Various experimental results for these three detectors, obtained on images taken by an on-board camera, are presented and analyzed in Section 6 . The last section is devoted to conclusions on the particular behavior of generative/discriminative features and their fusion. Some prospects are also presented. 


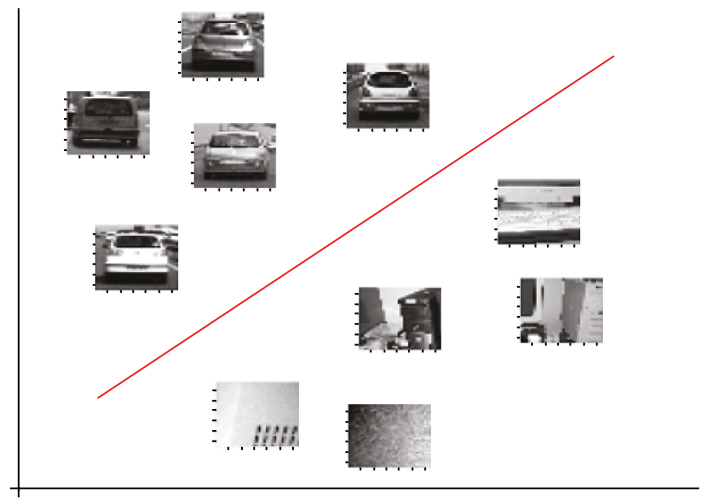

Feature space for discriminative classifier

(a)

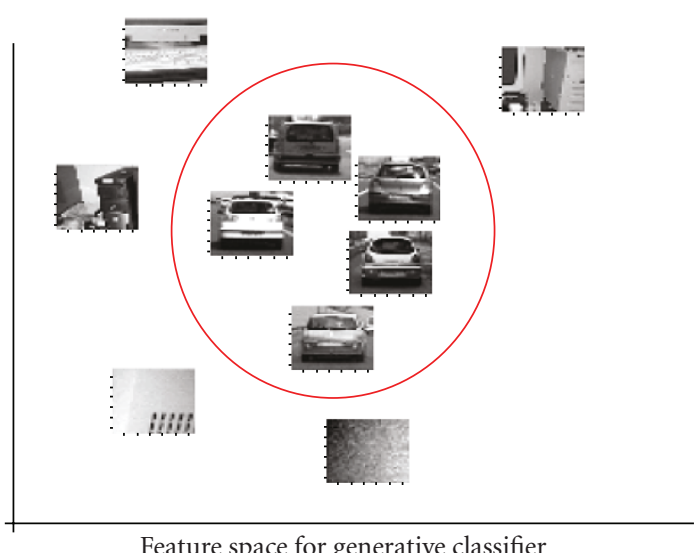

(b)

FIgURE 1: Feature space 2D.

\section{PREVIOUS WORKS}

A trivial solution for vehicle detection is the exhaustive search at all potential positions in the image. Of course, this solution is not satisfactory for real-time applications. To solve this problem, most of the methods reported in the literature follow an attentional process which can be decomposed into two steps [3] as follows.

(i) Hypotheses generation: the system provides potential positions of vehicles in a simple and rapid way resulting in a reduced search area.

(ii) Hypotheses validation: hypotheses generated in the earlier step are verified by using some complex algorithms and false alarms are eliminated.

Hypotheses generation is based on simple, low-level algorithms which estimate potential vehicle locations. They can be classified in three categories: knowledge-based (symmetry [4], color [5, 6], shadows [7], edges [8], corners [9], texture [10]), stereo-based (disparity map [11], inverse perspective mapping [12]), and motion-based [13].

The different approaches used for hypotheses validation are either model-based or appearance-based.

The first methods use a predefined template of the vehicle class and perform a correlation between the template and the input image for validation. Templates can be either rigid $[14,15]$ (horizontal or vertical contours [16]) or deformable [17-19].

Appearance-based methods learn the vehicle class characteristics from a set of images. Each training image is represented by a [20] feature vector. Then a classifier (neural network, support vector machine, Bayesian, etc.) is trained to estimate the decision boundary between the vehicle class and the nonvehicle class.

In addition to these methods, Viola and Jones [2] proposed an original attentional scheme for detection. The approach consists in a cascade of boosted classifiers with increasing complexity: each layer in the cascade reduces the search zone while rejecting regions that do not contain any object. This method uses Haar-like features, also called rectangular filters (experimented by Papageorgiou et al. $[21,22])$, and Adaboost learning [23]. The latter permits to select a limited number of features in each layer. In reality, Adaboost does not select the best features but the associated weak classifiers (cf. Section 4). The use of an integral image to calculate Haar-like features and the cascade approach results in a real-time face-detection application.

This approach has inspired a lot of recent works in vehicle detection. They propose some improvements about the used features $[3,24,25]$, their selection process $[24,25]$, the boosting algorithm (RealBoost [26], GentleBoost [27]), and the cascade architecture [27].

In this work, two types of features are employed and hence compared: rectangular filters (Haar-like features or Haar features) and HoG. These two features are frequently used in the domain of object detection and recognition.

Haar-like features were introduced by $[21,22]$ for pedestrian and vehicle detection. They are derived from the wavelet decomposition (using Haar wavelets). The filter set was enhanced by different works $[2,28]$, and does not comply strictly with the wavelet theory. Thus, they are called rectangular filters or Haar-like filters.

The histogram of oriented gradients is a histogram of neighbourhood pixels according to their gradient orientation and weighted by their gradient magnitude. Recently [29-31], HoG are used in a feature set called scale invariant feature $t$ ransform (SIFT) [32], which are employed successfully for pedestrian detection. In [29], the authors regrouped SIFT computed on a window and used a linear SVM for classification.

In [31], they used SIFT instead of Haar-like features in Viola and Jones detector. Linear SVM acting as weak learners are used in the cascade.

As we can see, in all previous approaches, HoG are used with discriminative classifiers. In this paper, we propose to associate generative classifiers with $\mathrm{HoG}$ features and to concatenate generative and discriminative features. Some papers have already proposed to concatenate two different 


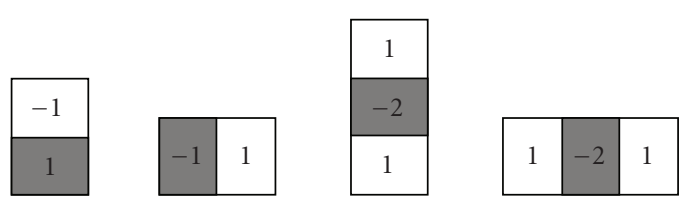

Figure 2: Set of Haar-like features.

features: Haar+HoG [30] or Haar+Gabor [33], but they only employ discriminative classifiers.

\section{FEATURE SPACE}

Positive examples (windows with vehicle) and negative examples (windows without vehicle) are distributed in an $N$ dimensional space depending on the features used to extract information. In the initial space (pixel grey-levels), classes may be overlapped. While choosing a good representation space and an adequate classifier, we can separate them. In our work, two types of features have been evaluated: Haar-like features and HoG. The former feature set defines a discriminative model of vehicles which separates the two classes by a decision boundary (hyper plane). The test examples will be categorised by their position in the feature space with respect to this hyperplane. The vehicle model estimated with the histograms of oriented gradients is generative. A class model is established from the training database, and the test examples are compared to the model and are categorised by using their dissimilarity. Now, we describe the adopted feature spaces for vehicle detection.

\subsection{Rectangular filters or Haar-like features}

Rectangular filters or Haar-like features provide information about the grey-level distribution of two adjacent regions in an image.

Figure 2 shows the set of Haar filters used in our algorithm. These filters consist of two or three rectangles. To compute the output of a filter on a certain region of image, the sum of all pixels values in the grey region is subtracted from the sum of all pixels values in the white one (and normalized by a coefficient in case of a filter with three rectangles).

Viola and Jones [2] introduced the integral image which is an intermediate representation of an input image and reduces the computation time for the filters. Sum of the rectangular regions can be calculated by using only four references in the integral image. As a result, the difference of two adjacent rectangular regions can be computed by using only six references in the integral image. For a filter with three rectangular regions, only eight references are needed. At the same time, integral image allows to perform fast variance normalization, necessary to reduce the effect of different lighting conditions.

Figure 3 illustrates the filtering of an image using two types of rectangular filters on two different scales: $1 \times 2$ and $2 \times 4$ pixels. These pictures show that the chosen filters emphasize the horizontal and vertical edges in the image. We

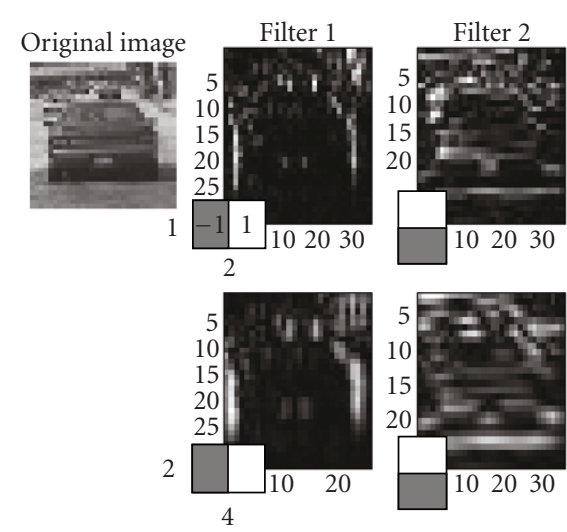

FIGURE 3: Original image and different image windows obtained by applying the vertical and horizontal Haar filters.

can also observe that when the filter size is doubled, details in the image are filtered while conserving the most important edges.

Every feature $j$ is defined as $f_{j}\left(x_{j}, y_{j}, s_{j}, r_{j}\right)$, where $r_{j}$ is the type of rectangular filter (see Figure 2), $s_{j}$ is the scale, and $\left(x_{j}, y_{j}\right)$ is its position in the window. Five scales are used for the two rectangles filters: $1 \times 2,2 \times 4,4 \times 8,8 \times 16,16 \times 32$ (similar scales are used for the three rectangles filters).

The Haar feature space is defined by a vector containing 8151 features for a window size of $32 \times 32$ pixels.

\subsection{Histogram of oriented gradient}

The other feature space used in this work is HoG. This local feature uses gradient magnitude and orientation around a point of interest or in a region of the image to construct the histograms. To calculate the input image (grey-level) gradient, we apply a Sobel filter of size $3 \times 3$. The orientation of pixels is then quantized to integer values between 0 and $N-1$ (here $N=4$ ) using modulo $\pi$ instead of modulo $2 \pi$.

Each histogram is computed as follows:

(i) all the pixels of the rectangular region are traversed;

(ii) for each pixel with gradient orientation $o$, the value of the corresponding bin is incremented by the gradient magnitude at the pixel (the number of quantized histogram bins is $N$ );

(iii) once all the pixels are evaluated, the bin values are normalised to obtain their sum equals to 1 .

The HoG feature space is defined by 3917 histograms computed in a rectangular area of $32 \times 32$ pixels. Each histogram $j$ is defined as $h_{j}\left(x_{j}, y_{j}, s_{j}, r_{j}\right)$, where $r_{j}$ is the type of rectangle, $s_{j}$ is the scale, and $\left(x_{j}, y_{j}\right)$ is its position in the window.

The types of rectangles depend on the width/height ratio which can be $(1 \times 1),(1 \times 2),(2 \times 1)$. We have a total of four scales: $s:\{2,4,8,16\}$.

We observe from the examples of Figure 4 that the majority of the contours found in a certain region are horizontal (bin two of the histogram). The other region 
contains the contours of all types but we can see a large number of vertical contours.

We use an intermediate representation (integral histogram [34]) of the input image (inspired in the integral image) which permits to rapidly compute the histograms. We obtain, in the similar way as we obtain with the integral image, a three-dimensional table (the third dimension corresponds to orientation) which allows us to accumulate gradient magnitude for a certain given orientation in a region with the help of four references in the integral histogram. In this way, the complete histogram can be built with $4 \times N$ references in the integral histogram.

\section{AdaBOOST}

The size of feature set is many times greater than the number of pixels in the input image. Keeping in view the computation time and robustness, the use of this much large set for classification is not suitable because some features from this set do not contain any useful information (noise). In literature, different methods have been used for the selection of useful and representative features: statistical methods [35], principal component analysis [36], genetic algorithms $[37,38]$, and so forth.

\subsection{Discrete Adaboost}

Among these methods, Adaboost algorithm [23] has shown its capability to improve the performance of various classification and detection systems. It finds precise hypotheses by combining several weak classification functions which, in general, have moderate precision. Adaboost is an iterative algorithm that finds, from a feature set, some weak but discriminative classification functions and combines them in a strong classification function:

$$
G= \begin{cases}1, & \sum_{t=1}^{T} \alpha_{t} g_{t} \geq \frac{1}{2} \sum_{t=1}^{T} \alpha_{t}=S, \\ 0, & \text { otherwise, }\end{cases}
$$

where $G$ and $g$ are the strong and weak classification functions, respectively, and $\alpha$ is a weight coefficient for each g. $S$ is the threshold of strong classifier $G$.

Different variants of boosting algorithm are developed: discrete AdaBoost [2], real AdaBoost [39], gentle AdaBoost, and so forth. However, we use the first one defined by Pseudocode 1.

To use this algorithm, we have to define the weak classifiers for two different types of features: Haar and HoG.

\subsubsection{Weak classifier-Haar}

We define the weak classifier for a feature $j$ as a binary response $g_{\text {Haar }}$ :

$$
g_{\text {Haar }}= \begin{cases}1, & \text { if } p_{j} f_{j}<p_{j} \theta_{j}, \\ 0, & \text { otherwise, }\end{cases}
$$

where $f_{j}$ is the absolute value of the feature $j$ and $\theta_{j}$ is the threshold, and $p_{j}$ is the parity. For each feature $j$,
AdaBoost determines an optimal threshold $\theta_{j}$ for which the classification error on training database (with positive and negative examples) is minimised.

\subsubsection{Weak classifier-HoG}

In this case, we construct a generative classifier based on the class (vehicle) model. The median of histograms of positive examples from training database is used as our model, defined as

$$
m_{j}=\operatorname{median}\left\{h_{j}^{i}\right\}_{i=1, \ldots, P}
$$

where $P$ is the number of positive examples in the training database.

The generative classifier computes the distance between a histogram $h_{j}$ of the input image and a model histogram $m_{j}$.

We defined the weak classifier $g_{\mathrm{HoG}}$ as follows

$$
g_{\mathrm{HoG}}= \begin{cases}1, & \text { if } d\left(h_{j}, m_{j}\right)<\theta_{j}, \\ 0, & \text { otherwise, }\end{cases}
$$

where $d\left(h_{j}(x), m_{j}\right)$ is the Bhattacharya distance between the histogram $h_{j}$ and model histogram $m_{j}$, and $\theta_{j}$ is the optimal threshold on the distance for this feature.

Bhattacharya distance is defined as

$$
d\left(h_{j}, m_{j}\right)=\sqrt{1-h_{j} \bullet m_{j}}
$$

where [•] is the scalar product.

The distance is a similarity measure between two histograms, that is, values close to 0 for similar histograms. The output values are bounded between 0 and 1 .

\section{IMPLEMENTATION}

In this section, we describe the image database used for the training and for the test. Later, we present our implementation.

\subsection{Database}

The database used for experimentation contains more than 557 images of one or more rear viewed vehicles, resulting in more than 1500 vehicle images of typical cars, sport-utility vehicles (SUVs), and minivans. The dataset was labelled manually by enclosing each vehicle in a bounding box.

We have constructed three databases as follows.

(i) Vehicle database: the positive database contains 745 examples. The number of images is doubled by synthesizing a mirror image (along the vehicle axis of symmetry). From a total of 1490 vehicle images, twothirds are used as positive training set and one-third as positive validation set. This validation set is used to tune the strong classifier decision threshold $S$ to reach the minimum acceptable correct detection rate $\left(\mathrm{DR}_{\min }\right)$ and the maximum acceptable false-alarm rate $\left(\mathrm{FA}_{\max }\right)$ during cascade training (see Section 5.3). This validation set is independent of the positive training 

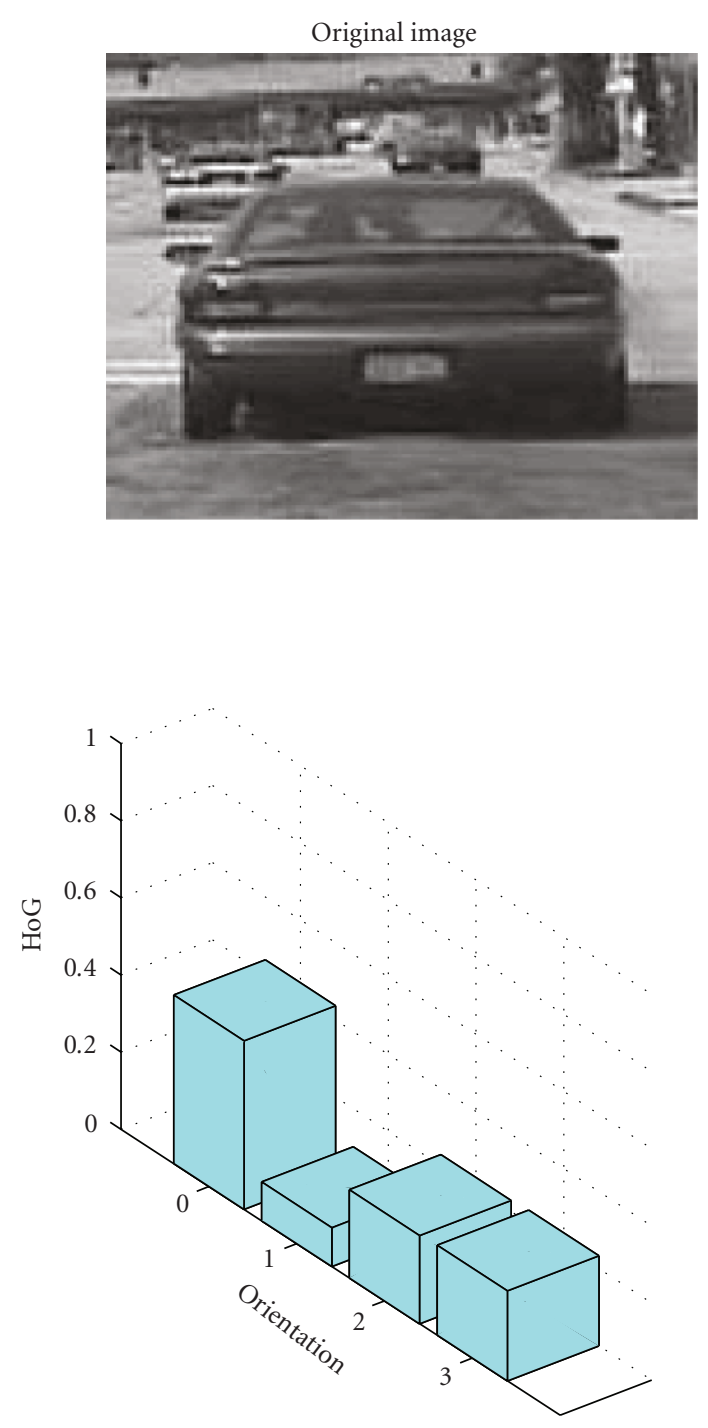
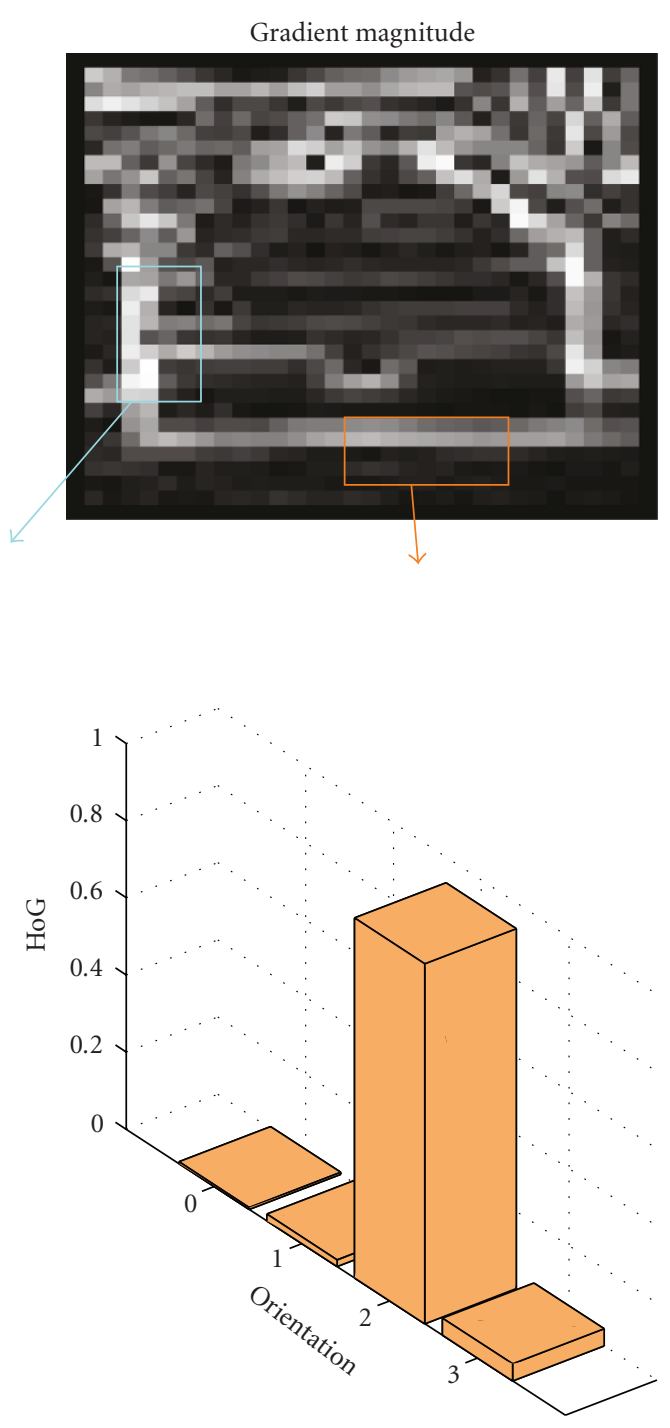

Figure 4: Result of application of HoG on a vehicle image.

set used to select weak classifiers. Some positive examples from the training set are shown in Figure 5.

(ii) Test database: composed of 230 on-road scene images containing 472 vehicles.

(iii) Negative database: composed of negative examples which are taken randomly in a set of more than 4000 arbitrary images (which do not contain any vehicle).

During training, the size of the smallest window used is $32 \times 32$ pixels. It also corresponds to the minimum size of an object that can be detected in an image. In our case, it corresponds to a vehicle at nearly 80 meters apart from the vehicle carrying the vision system.

\subsection{Single detector}

A single detector is a strong classifier $G$ (i.e., without cascade) composed of $T$ features or weak classifiers and trained using Adaboost algorithm. Three detectors are constructed according to the choice of features. The first two are trained by using individual features of Haar and HoG. The third one is trained on the concatenation of both Haar and HoG features. Five thousand (5000) windows are used in the negative database for training.

To evaluate the performance of the training method, we employ cross-validation. We obtained three classifiers from three different training databases: positive examples in training and cross-validation databases are chosen randomly. In the same way, the negative examples are drawn randomly to construct the negative database. The correct detections rate (DR), evaluated on test database and used in Section 6, is the average of DR on all the three detectors. In the same way, we used the average of false alarms (FAs).

\subsection{Cascade detector}

In this section, we discuss the implementation of the attentional cascade [2]. This architecture had shown to be an appropriate method for fast and reliable object detection 
(1) Given $N$ examples $\left(x_{1}, y_{1}\right), \ldots,\left(x_{N}, y_{N}\right)$ with $x \in \mathfrak{R}$ and $y_{i} \in\{0,1\}$

(2) Initialise $w_{i}=1 / N, i=1, \ldots, N$

(3) For $t=1, \ldots, T$

For each feature $j$, train a classifier $g_{j}$ using $w_{i}$ for which the error is defined as: $\epsilon_{j}=\sum_{i=1} \omega_{i}\left|g_{j}\left(x_{i}\right)-y_{i}\right|$

Choose a classifier $g_{t}$ with lowest error $\epsilon_{t}$ Update weights: $\omega_{t+1, i}=\omega_{t, i} \beta_{t}^{1-e_{i}}$

where $e_{i}=0$ if $g_{t}\left(x_{i}\right)=y_{i}, e_{i}=1$ otherwise, with $\beta_{t}=\epsilon_{t} /\left(1-\epsilon_{t}\right)$

(4) Output: $\left.G=\sum_{t=1}^{T} \alpha_{t} g_{t} \geq\right)(1 / 2) \sum_{t=1}^{T} \alpha_{t}$ with $\alpha_{t}=\log \left(1 / \beta_{t}\right)$

Pseudocode 1: Discrete AdaBoost.

on embedded hardware $[25,40]$. The cascade is composed of a series of strong classifiers $G_{i}$. Each strong classifier in the cascade is trained using AdaBoost. Instead of stopping the iterative process according to a maximum number of features $T$, we fix two performance parameters of strong classifier $G_{i}$ : the minimum acceptable correct detections rate $\mathrm{DC}_{\min }$ and maximum acceptable false-alarm rate $\mathrm{FA}_{\max }$.

The negative database $N_{i}$, used for training the strong classifier $G_{i}$ at layer $i$, is formed of those negative examples which were misclassified (categorized as vehicles) by the preceding layers.

Till now, we have defined three stopping criteria for the cascade training as follows.

(1) The first is the maximum number of training iterations (limited to 200) for strong classifier $G_{i}$ without reaching the maximum acceptable false-alarm rate or minimum acceptable rate of correct detections. We observed from the results that, for numerous cascades (called Non Conv), the algorithm has not converge in the last stage.

(2) The cascade gets a global false-alarm rate lower than the objective (called $F$ attained). Here, the objective is $F=43 * 10^{-7}$, obtained for 16 stage cascade with a $\mathrm{FA}_{\max }$ equal to $40 \%$.

(3) It is not possible to find sufficient number of negative examples (called non-Neg).

The choice of $\mathrm{DC}_{\min }$ and $\mathrm{FA}_{\max }$ modifies the cascade behavior and their architecture. The algorithm given in [2] indicates that the threshold $S_{i}$ of the strong classifier $G_{i}(1)$ is decreased until $G_{i}$ has a detection rate of at least $\mathrm{DC}_{\min }$ on the validation set. The higher the $\mathrm{DC}_{\min }$, the lower the $S_{i}$, and the vehicle model will perform better on difficult positives. On the other hand, more negative examples will be considered as positives (false alarms). The value of $\mathrm{DC}_{\min }$ also gives the detection rate for the attentional cascade: $D_{\mathrm{AC}}=\left(\mathrm{DC}_{\min }\right)^{K}$, where $K$ is the number of layers in the cascade. For example, the choice of $\mathrm{DC}_{\min }=99.5 \%$ achieves a detection rate of $92.3 \%$ for a 16 -stage classifier.
Considering the classifier threshold is decremented to reach $D C_{\text {min }}$, the iterative process will be stopped when the strong classifier $G_{i}$ does not exceed $\mathrm{FA}_{\max }$ on the negative dataset. Taking into account the cascade would reject off at least one half of negatives at each layer, $\mathrm{FA}_{\max }$ can be $50 \%$. To get lower values of $\mathrm{FA}_{\max }, G_{i}$ needs weaker classifiers. In these cases, the global false-alarm rate $F$ can be achieved shortly, obtaining a cascade with a reduced number of layers.

We obtained three different detectors trained on the three feature spaces: Haar-like features, HoG, and their fusion. Three versions of each detector are realised by varying the number of negative examples used during training: 1000, 2000, 3000 negative examples.

\subsection{Controlled cascade}

Without any kind of supervision, the training of the different feature spaces results in dissimilar cascade architectures: features by layer and number of layers (Figure 7). In order to obtain comparable cascade detectors and, at the same time, to avoid the nonconvergences (as frequently observed, see Table 2), we modify the training process by changing the criterion used to stop the training of the strong classifier $G_{i}$. For a certain stage in the cascade, if an upper bound on the maximum number of features is reached without converging (i.e., it does not achieve $\mathrm{DR}_{\min }$ and $\mathrm{FA}_{\max }$ ), the iterative process is stopped and the function $G_{i}$ is conserved in this state. Then the function $G_{i+1}$ of the next stage is trained.

To fix this upper bound in each stage of the cascade, we use an exponential law. This choice is based on the fact that we only need a small quantity of features in the earlier stages of the cascade to eliminate "easy" examples (far from the boundary). When we move further, the number of features must be increased as the later stages have to face more complex examples (positive and negative examples are near the boundary and are hard to separate). So our choice of exponential law serves this task and helps us in finding an appropriate number of features for each layer.

\section{RESULTS}

In this section, we analyse the results obtained for three different types of detectors (Haar, HoG, and fusion) and for the three different implementations (simple, cascade, and controlled cascade). The performance measures are the correct detections rate corresponding to the ratio of correct detections to the total number of vehicles present in the test database: the false-alarm rate computed as the average number of false alarms per image (calculated on all the test images) divided by the total number of windows evaluated by the detector in an image. In total, 31514 windows are evaluated in every image at various positions and at different scales. Detected rectangles are considered as hits if they fulfil a coincidence criterion with the reference positive bounding box. The parameters used are a maximum difference size and a maximum difference position, with values 1.5 and 0.3 , respectively (OpenCV method). The average computation time for an image is evaluated on a PC with $2.2 \mathrm{GHz}$ processor. 

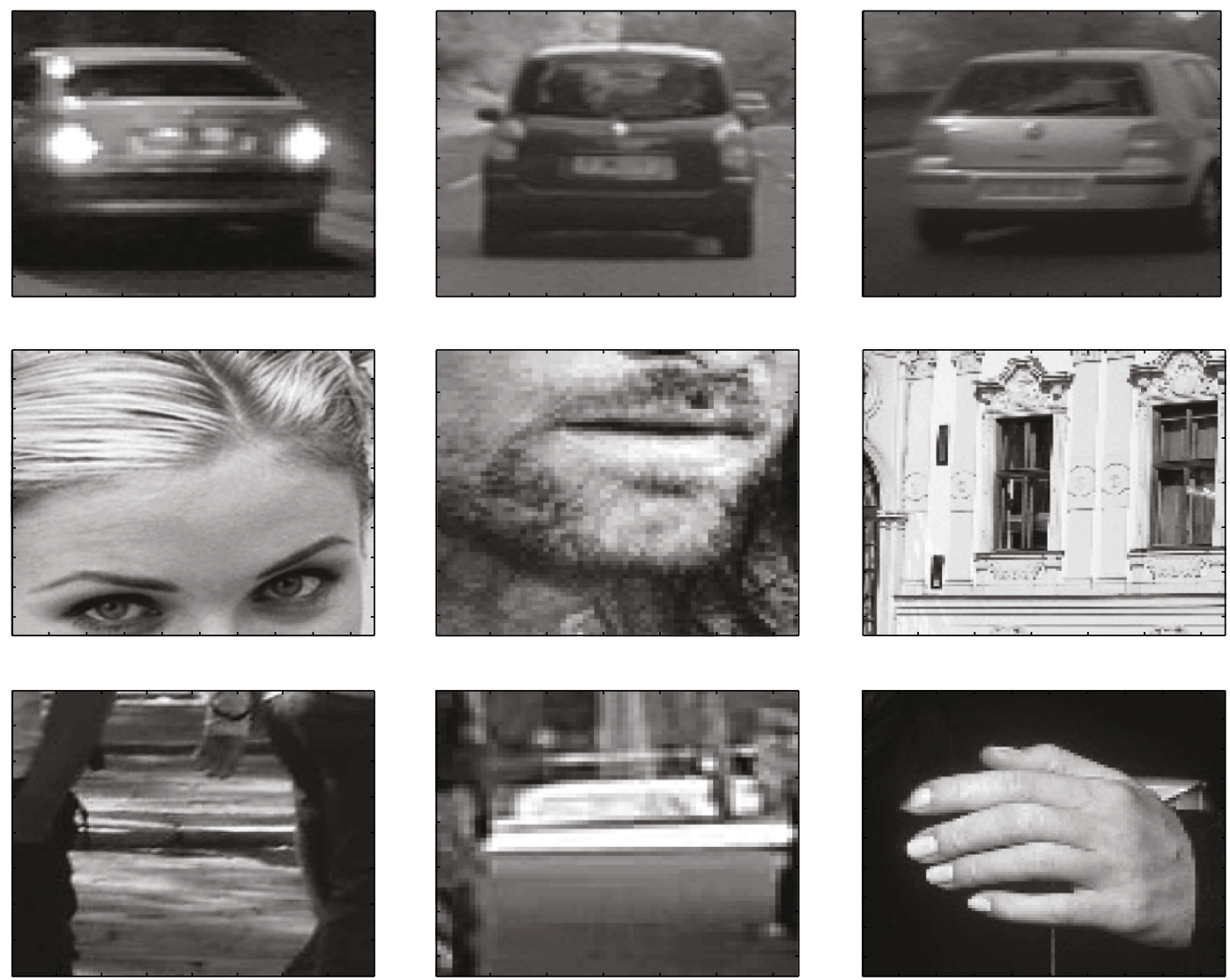

FIgUre 5: Positive and negative examples used for training. The first line shows positive examples (vehicle). The other lines show negative examples (nonvehicle) used to train the attentional cascade: easy examples used in first stages for the second line and difficult examples used in the last stages for the third line.

\subsection{Single detector}

For each detector, we varied the number of features $(T=$ 50, 100, and 150 features). Figure 6 shows the ROC curves for each detector (Haar, HoG, and fusion) obtained by changing the threshold $S$ of (1).

In this figure, we observe the following:

(i) to obtain a low false-alarm rate (less than 0.005), Haar detector performs better than HoG detector,

(ii) inversely, for a high false-alarm rate, fusion detector provides a higher correct detection rate than Haar detector,

(iii) HoG detector behaves in a similar manner as fusion detector but with a larger quantity of false alarms.

These conclusions are confirmed in Table 1 which details the performances of the single detectors when the threshold is tuned to obtain a global correct detection rate greater than $99.5 \%$ on the positive validation database.

Increasing the number of features refines the decision boundary for Haar features and the model for HoG features. Comparing the false-alarm rate, Haar-like features are more discriminative than HoG features. The fusion of these two gives intermediate results while conserving a high detection rate and eliminating a large number of false alarms.
TABLE 1: Table of results for single detectors.

\begin{tabular}{lcccc}
\hline Type & no. Desc & DR $(\%)$ & FA & Time $(\mathrm{sec})$ \\
\hline Haar & 50 & 99.8 & 0.0220 & 1.42 \\
Haar & 100 & 99.8 & 0.0145 & 3.51 \\
Haar & 150 & 99.0 & 0.0044 & 5.17 \\
\hline HoG & 50 & 100 & 0.0588 & 0.90 \\
HoG & 100 & 99.9 & 0.0300 & 1.68 \\
HoG & 150 & 99.9 & 0.0233 & 2.33 \\
\hline Fusion & 50 & 99.6 & 0.0130 & 1.67 \\
Fusion & 100 & 99.3 & 0.0093 & 3.19 \\
Fusion & 150 & 99.2 & 0.0063 & 4.75 \\
\hline
\end{tabular}

We also observed that the computation time increases with the increase in the number of features. For realtime application, the use of a large number of features is unrealistic, so we have to adopt the cascade architecture.

\subsection{Cascade detector}

Table 2 details the architecture and the performances of each cascade detector. We used the following parameters of accuracy for the function $G_{i}$ : the minimum acceptable correct detection rate $\mathrm{DR}_{\min }=0.995$ and the maximum 


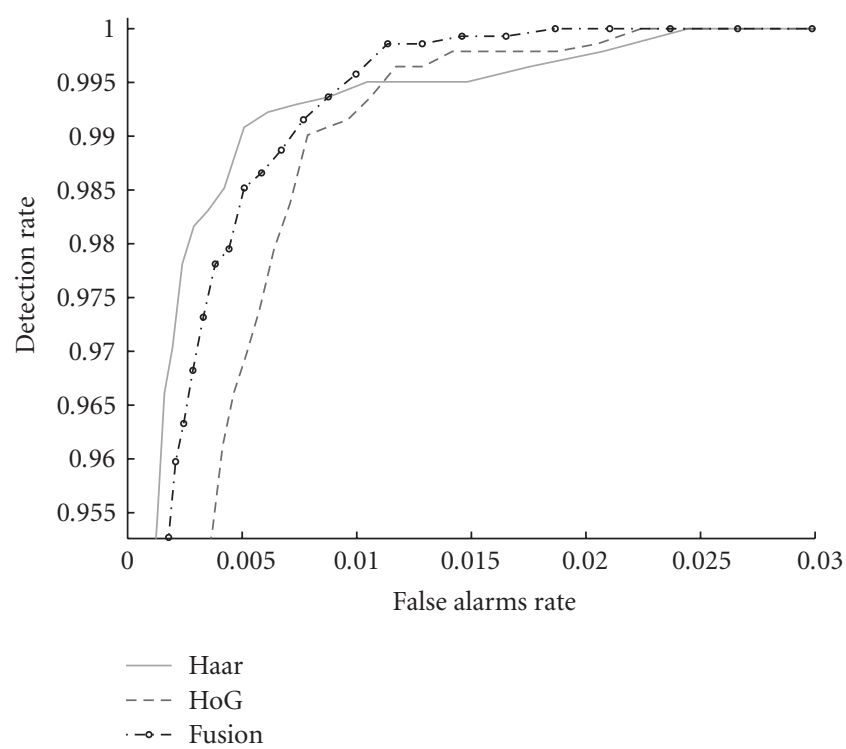

Figure 6: ROC curves of single detectors based on Haar, HoG, and fusion.

acceptable false-alarm rate $\mathrm{FA}_{\max }=0.40$. We observe a mismatch of number of layers between three detectors: in most cases, the algorithm AdaBoost does not converge.

Increasing the number of negative training examples increases the number of stages to achieve convergence. This can easily be explained as follows: a large number of negative examples allows to generate a robust model or a robust decision boundary in order to eliminate a huge number of false alarms in the early stages of the cascade; and very soon, the difficult cases are left for elimination, resulting in the nonconvergence of the algorithm.

Moreover, in the case of HoG detector, the number of features increases quickly (Figure 7). This behaviour is quite symptomatic of generative classifiers. They can model accurately positive examples (resulting in a high correct detection rate in Table 2), but they need to construct a rather complex model in order to fit an accurate decision boundary for those negative examples which are close to positive ones. It is also noteworthy that HoG strong classifiers converge with a small number of features in the early stages of the cascade as compared to Haar one. In fact, a small number of features are needed to eliminate those negative windows relatively far from the model and are sufficient for convergence. Inversely, Haar detector needs a lot of features to estimate correctly the decision boundary between the classes.

We can again observe that the combination of two features in fusion detector enhances the performance: it uses HoG features to eliminate those negative examples which are far from the model and uses Haar features to eliminate those near the decision boundary. Moreover, the cascade realisations do not have same number of layers as can be seen in Table 2.

We verified that the number of false alarms is strongly related to number of stages in the cascade. A large number

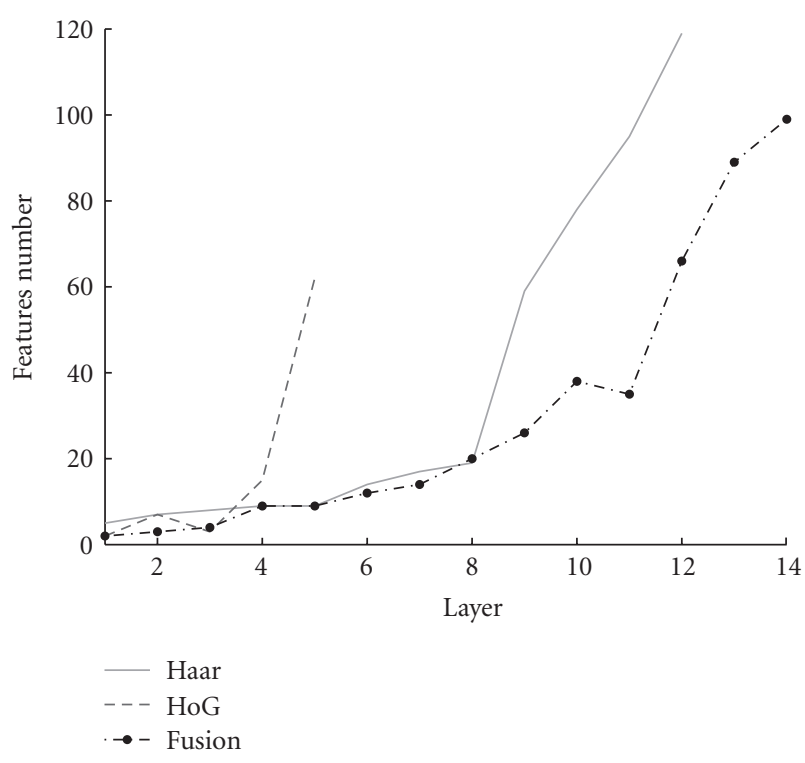

FIGURE 7: Number of features retained by each layer for a detector with 1000 negative examples.

of stages can eliminate a lot of false alarms. However the number of correct detections may also get reduced. We will see that controlling the number of features per stages during training, as proposed in Section 5.4, will allow us to increase the number of stages and to enhance the performance of detectors. Moreover, with this approach, we will obtain the same number of stages for all detectors and hence will validate our above-mentioned hypotheses.

\subsection{Controlled cascade detector}

Figure 8 shows the number of features per stage for the detectors trained with 1000 negative examples. When a point is under the exponential curve, this means that the strong classifier has achieved its goal or has converged without exceeding the allowed number of features for this layer.

The HoG detector converged with a small number of features without reaching the maximum number of features in the earlier stages. On the other hand, in later stages, it was saturated and could not converge.

The Haar detector did not converge in first stages, but from the tenth stage (or later) it did. The fusion detector has an intermediate behavior that lies between the above two detectors. Figure 9 illustrates the evolution of the proportion of HoG features with respect to the total number of features chosen at each layer. We observe that in the initial stages, HoG features are chosen as being more discriminative, while in later layers, strong classifiers are mainly based on Haar features.

This confirms our previous hypotheses for cascade detectors without features controlling mechanism. Moreover, this can be deduced from Table 3 which summarizes the global performance of controlled cascade detectors. The HoG detector obtains a high correct detections rate while the number of false alarms is also high. 
TABLE 2: Table of results for the cascade detectors.

\begin{tabular}{lccccccc}
\hline Type & no. Neg & no. Layers & no. Desc & DR $(\%)$ & FA & Time & Stop \\
\hline Haar & 1000 & 12 & 430 & 479 & 95.4 & 0.00080 & 0.59 \\
Haar & 2000 & 11 & 272 & 96.4 & 0.00070 & 0.57 & Non-Conv \\
Haar & 3000 & 10 & 89 & 97.7 & 0.00099 & 0.58 & Non-Conv \\
\hline HoG & 1000 & 5 & 52 & 99.8 & 0.030 & 0.73 & Non-Conv \\
HoG & 2000 & 5 & 21 & 99.9 & 0.034 & 0.56 & Non-Conv \\
HoG & 3000 & 4 & 392 & 99.9 & 0.077 & 0.43 & Non-Conv \\
\hline Fusion & 1000 & 14 & 369 & 94.5 & 0.00027 & 0.39 & F Attained \\
Fusion & 2000 & 12 & 358 & 94.3 & 0.00035 & 0.37 & Non-Conv \\
Fusion & 3000 & 12 & & & 0.00039 & 0.36 & Non-Neg \\
\hline
\end{tabular}

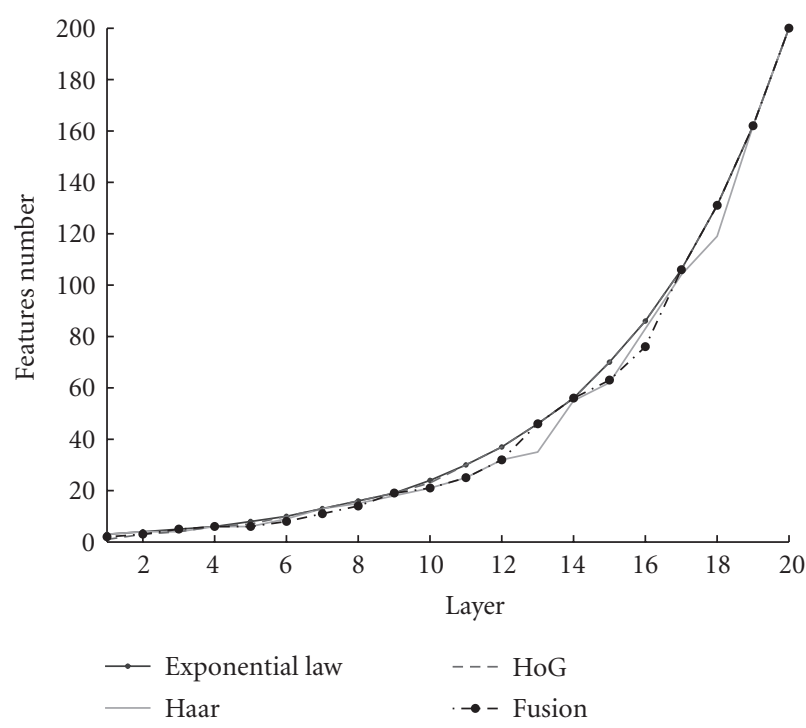

FIgURE 8: Number of features for the three detectors.

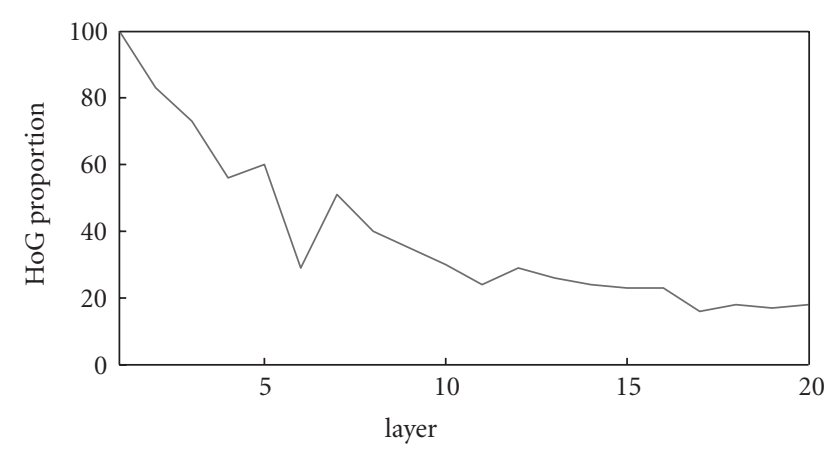

Figure 9: Proportion of chosen HoG features for each layer in the cascade for fusion detector.

Haar detector behaves inversely. It obtains a small quantity of false alarms at the end of the cascade, but a large number of positive examples were eliminated in the preceding layers (Figure 10). This figure describes the evolution of both detection rate and false-alarm rate with respect to the number of stages used. Figure 9 illustrates the fact that the fusion detectors combines the advantages of
TABLE 3: Table of results for controlled cascade detectors.

\begin{tabular}{lccccc}
\hline Type & no. Neg & no. Desc & DR $(\%)$ & FA & $t(\mathrm{~s})$ \\
\hline Haar & 1000 & 1016 & 93.8 & 0.00031 & 0.66 \\
Haar & 3000 & 942 & 89.83 & 0.00018 & 0.69 \\
\hline HoG & 1000 & 1027 & 97.8 & 0.0045 & 0.51 \\
HoG & 3000 & 1031 & 99.6 & 0.0114 & 1.07 \\
\hline Fusion & 1000 & 1022 & 94.0 & 0.00029 & 0.36 \\
Fusion & 3000 & 1021 & 93.5 & 0.00032 & 0.40 \\
\hline
\end{tabular}

the two features, generative for $\mathrm{HoG}$ and discriminative for Haar. In initial layers, it has essentially used the generative features to eliminate the negative examples far from model while conserving a high detection rate. In later layers, it used the discriminative features to generate fine decision boundary between positive examples and those negative examples which are near the model.

This is correctly reflected in on-road scene images in Figure 11, where white squares indicate (correct or false) detections. We observe that Haar detector does not detect all the vehicles but it does not produce many false alarms. On the contrary, HoG detector produces a large number of false alarms but detects all the vehicles. Fusion detector reduces the number of false alarms while detecting all the vehicles as in HoG case.

Hence the fusion detector performs much better compared to the two others, particularly considering the computing time. This can be explained easily by analysing the curve in Figure 10(b) where a large number of hypotheses are rejected by the fusion detector in the earlier stages of the cascade.

\section{CONCLUSIONS}

In this paper, we present a cascade of boosted classifiers for vehicle detection in on-road scene images. Two feature spaces have been evaluated: Haar-like features and HoG features. Haar-like features are used to construct discriminative weak classifiers while the other ones are used to construct generative weak classifiers. A third detector is obtained by concatenating these two feature vectors. 


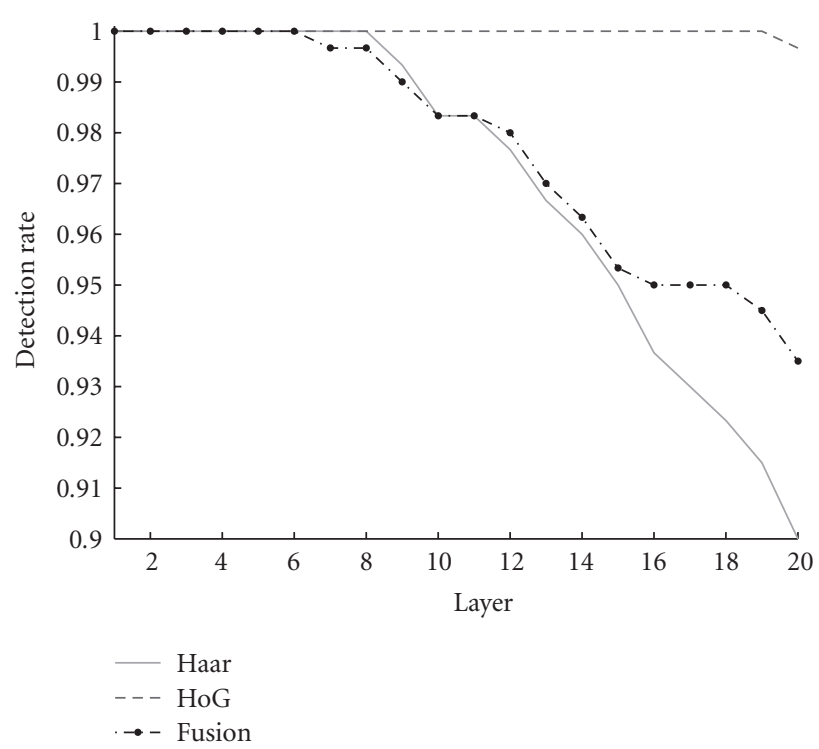

(a) Rate of correct detections per layer

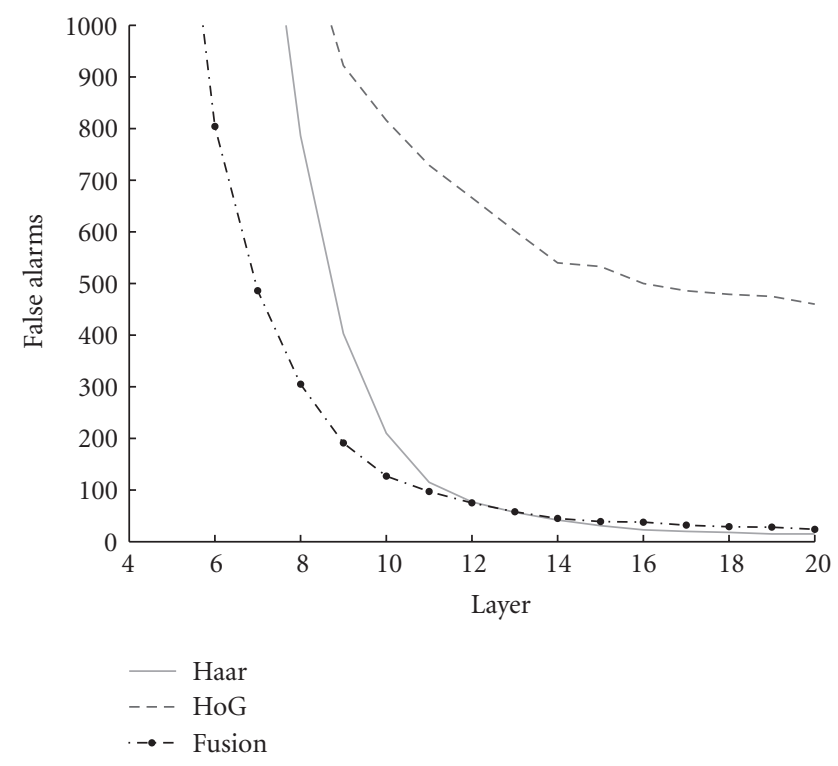

(b) Quantity of false alarms per layer

FIGURE 10: Behaviour of detection as a function of cascade for a detector with 3000 negative examples.
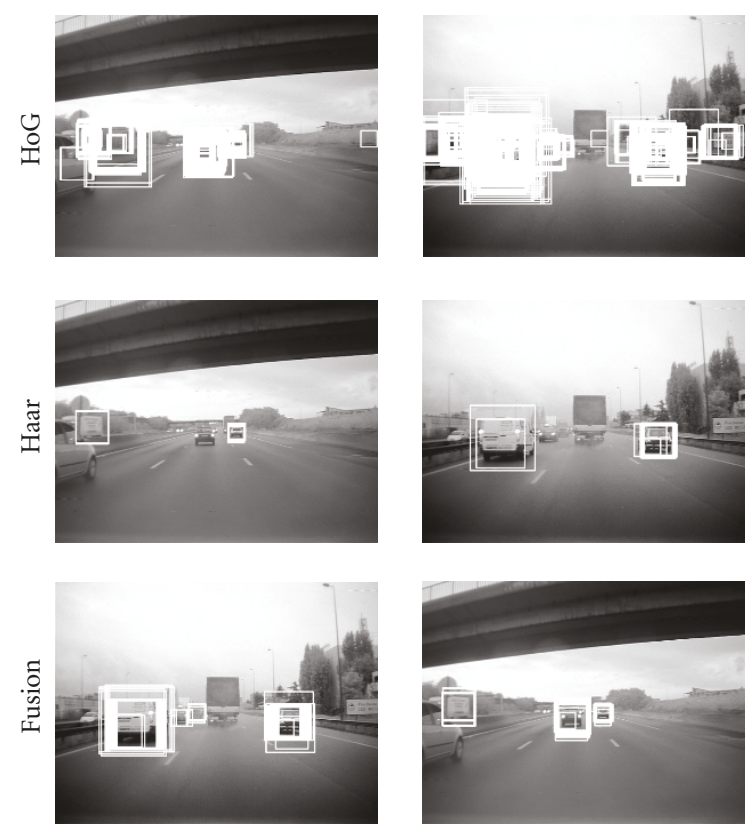

FIGURE 11: Detection results on road (motorway) scene images for three types of detectors.

We have studied the behavior of different architectures: single detector, cascade detector. To optimise the performance of cascade detector, we fix the maximum number of features per stage of the cascade, that is, for each strong classification function.

The fusion detector combines the advantages of both Haar and HoG detectors and achieves a high correct detection rate and a small number of false alarms. It uses the generative classifiers to eliminate those negative examples that are far from the model and then it employs discriminative classifiers to generate fine decision boundary between positive examples and those negative examples which are near to the model.

The main goal of this article is to show the complementarity between generative and discriminative classifiers. This complementarity was already proved-theoretically and experimentally-in [41-43]. This study demonstrates experimentally that the boosting process selects automatically in first place generative classifiers and then discriminative ones. Previously, this was done intuitively by combining sequentially both classifiers.

Our future work will be devoted to the use of these features to associate a classification system to this vehicle detection system. The classification system will categorise the several classes of the vehicle type: passenger car, light truck, van, and bus.

\section{ACKNOWLEDGMENTS}

This research is funded by Peugeot Citroën Automobile (PCA). The authors would like to thank M. Fabien Hernandez, from the PCA's Direction de la Recherche et de l'Innovation Automobile, for his support.

\section{REFERENCES}

[1] S. Han, E. Ahn, and N. Kwak, "Detection of multiple vehicles in image sequences for driving assistance system," in Proceedings of the International Conference on Computational Science and Its Applications (ICCSA '05), vol. 3480, pp. 11221128, Singapore, May 2005.

[2] P. A. Viola and M. J. Jones, "Robust real-time face detection," in Proceedings of the 8th IEEE International Conference on 
Computer Vision (ICCV '01), vol. 2, p. 747, Vancouver, BC, Canada, July 2001.

[3] Z. Sun, G. Bebis, and R. Miller, "On-road vehicle detection: a review," IEEE Transactions on Pattern Analysis and Machine Intelligence, vol. 28, no. 5, pp. 694-711, 2006.

[4] A. Bensrhair, M. Bertozzi, A. Broggi, P. Miche, S. Mousset, and G. Toulminet, "A cooperative approach to vision-based vehicle detection," in Proceedings of the 4th IEEE Conference on Intelligent Transportation Systems (ITSC '01), pp. 207-212, Oakland, Calif, USA, August 2001.

[5] D. Guo, T. Fraichard, M. Xie, and C. Laugier, "Color modeling by spherical influence field in sensing driving environment," in Proceedings of IEEE Intelligent Vehicles Symposium (IVS '00), pp. 249-254, Dearbon, Mich, USA, October 2000.

[6] T. Xiong and C. Debrunner, "Stochastic car tracking with lineand color-based features," IEEE Transactions on Intelligent Transportation Systems, vol. 5, no. 4, pp. 324-328, 2004.

[7] M. B. van Leeuwen and F. C. A. Groen, "Vehicle detection with a mobile camera," Tech. Rep., Computer Science Institute, University of Amsterdam, Amsterdam, The Netherlands, October 2001.

[8] F. Dellaert, "Canss: a candidate selection and search algorithm to initialize car tracking," Tech. Rep. CMU-RI-TR-97-34, Robotics Institute, Carnegie Mellon University, Pittsburgh, Pa, USA, 1997.

[9] M. Bertozzi, A. Broggi, and S. Castelluccio, "A real-time oriented system for vehicle detection," Journal of Systems Architecture, vol. 43, no. 1-5, pp. 317-325, 1997.

[10] T. Bucher, C. Curio, J. Edelbrunner, et al., "Image processing and behavior planning for intelligent vehicles," IEEE Transactions on Industrial Electronics, vol. 50, no. 1, pp. 62-75, 2003.

[11] U. Franke and A. Joos, "Real-time stereo vision for urban traffic scene understanding," in Proceedings of IEEE Intelligent Vehicles Symposium (IVS '00), pp. 273-278, Dearbon, Mich, USA, October 2000.

[12] M. Bertozzi and A. Broggi, "Vision-based vehicle guidance," Computer, vol. 30, no. 7, pp. 49-55, 1997.

[13] C. Demonceaux, A. Potelle, and D. Kachi-Akkouche, "Obstacle detection in a road scene based on motion analysis," IEEE Transactions on Vehicular Technology, vol. 53, no. 6, pp. 16491656,2004

[14] T. N. Tan and K. D. Baker, "Efficient image gradient based vehicle localization," IEEE Transactions on Image Processing, vol. 9, no. 8, pp. 1343-1356, 2000.

[15] H. Yang, J. Lou, H. Sun, W. Hu, and T. Tan, "Efficient and robust vehicle localization," in Proceedings of IEEE International Conference on Image Processing (ICIP '01), vol. 2, pp. 355-358, Thessaloniki, Greece, October 2001.

[16] N. Srinivasa, "Vision-based vehicle detection and tracking method for forward collision warning in automobiles," in Proceedings of the IEEE Intelligent Vehicle Symposium (IV'02), vol. 2, pp. 626-631, Versailles, France, June 2002.

[17] J. M. Collado, C. Hilario, A. de la Escalera, and J. M. Armingol, "Model based vehicle detection for intelligent vehicles," in Proceedings of IEEE Intelligent Vehicles Symposium (IVS '04), pp. 572-577, Parma, Italy, June 2004.

[18] M.-P. Dubuisson Jolly, S. Lakshmanan, and A. K. Jain, "Vehicle segmentation and classification using deformable templates," IEEE Transactions on Pattern Analysis and Machine Intelligence, vol. 18, no. 3, pp. 293-308, 1996.

[19] N. H. C. Yung and A. H. S. Lai, "Detection of vehicle occlusion using a generalized deformable model," in Proceedings of the IEEE International Symposium on Circuits and Systems (ISCAS '98), vol. 4, pp. 154-157, Monterey, Calif, USA, MayJune 1998.

[20] S. Agarwal and D. Roth, "Learning a sparse representation for object detection," in Proceedings of the 7th European Conference on Computer Vision (ECCV '02), pp. 113-130, Springer, Copenhagen, Denmark, May 2002.

[21] M. Oren, C. Papageorgiou, P. Sinha, E. Osuna, and T. Poggio, "Pedestrian detection using wavelet templates," in Proceedings of the IEEE Computer Society Conference on Computer Vision and Pattern Recognition (CVPR '97), pp. 193-199, San Juan, Puerto Rico, USA, June 1997.

[22] C. Papageorgiou and T. Poggio, "A trainable system for object detection," International Journal of Computer Vision, vol. 38, no. 1, pp. 15-33, 2000.

[23] Y. Freund and R. E. Schapire, "Experiments with a new boosting algorithm," in Proceedings of the 13th International Conference on Machine Learning (ICML '96), pp. 148-156, Bari, Italy, July 1996.

[24] Y. Abramson, F. Moutarde, B. Steux, and B. Stanciulescu, "Combining adaboost with a hill-climbing evolutionary feature-search for efficient training of performant visual object detectors," in Proceedings of the 7th International FLINS Conference on Applied Artificial Intelligence (FLINS '06), Genova, Italy, August 2006.

[25] B. Alefs, "Embedded vehicle detection by boosting," in Proceedings of the 9th IEEE International Conference on Intelligent Transportation Systems (ITSC '06), pp. 536-541, Toronto, Ontario, Canada, September 2006.

[26] D. Ponsa and A. Lopez, "Cascade of classifiers for vehicle detection," in Proceedings of the 9th International Conference on Advanced Concepts for Intelligent Vision Systems (ACIVS '07), vol. 4678, pp. 980-989, Delft, the Netherlands, August 2007.

[27] D. Withopf and B. Jahne, "Improved training algorithm for tree-like classifiers and its application to vehicle detection," in Proceedings of the IEEE International Conference on Intelligent Transportation Systems (ITSC '07), pp. 642-647, Seattle, Wash, USA, September 2007.

[28] R. Lienhart, A. Kuranov, and V. Pisarevsky, "Empirical analysis of detection cascades of boosted classifiers for rapid object detection," in Proceedings of the 25th Pattern Recognition Symposium (DAGM '03), vol. 2781, pp. 297-304, Magdeburg, Germany, September 2003.

[29] N. Dalal and B. Triggs, "Histograms of oriented gradients for human detection," in Proceedings of the IEEE Computer Society Conference on Computer Vision and Pattern Recognition (CVPR '05), vol. 1, pp. 886-893, San Diego, Calif, USA, June 2005.

[30] D. Gerónimo, A. López, D. Ponsa, and A. D. Sappa, "Haar wavelets and edge orientation histograms for on-board pedestrian detection," in Proceedings of the 3rd Iberian Conference on Pattern Recognition and Image Analysis (IbPRIA '07), pp. 418425, Girona, Spain, June 2007.

[31] Q. Zhu, M.-C. Yeh, K.-T. Cheng, and S. Avidan, "Fast human detection using a cascade of histograms of oriented gradients," in Proceedings of the IEEE Computer Society Conference on Computer Vision and Pattern Recognition (CVPR '06), vol. 2, pp. 1491-1498, New York, NY, USA, June 2006.

[32] D. G. Lowe, "Object recognition from local scale-invariant features," in Proceedings of the 7th IEEE International Conference on Computer Vision (ICCV '99), vol. 2, pp. 1150-1157, Kerkyra, Greece, September 1999. 
[33] Z. Sun, G. Bebis, and R. Miller, "On-road vehicle detection using evolutionary gabor filter optimization," IEEE Transactions on Intelligent Transportation Systems, vol. 6, no. 2, pp. 125-137, 2005.

[34] F. Porikli, "Integral histogram: a fast way to extract histograms in cartesian spaces," in Proceedings of the IEEE Computer Society Conference on Computer Vision and Pattern Recognition (CVPR '05), vol. 1, pp. 829-836, San Diego, Calif, USA, June 2005.

[35] H. Schneiderman and T. Kanade, "A statistical method for 3D object detection applied to faces and cars," in Proceedings of the IEEE Computer Society Conference on Computer Vision and Pattern Recognition (CVPR '00), vol. 1, pp. 746-751, Hilton Head Island, SC, USA, June 2000.

[36] J. Wu and X. Zhang, "A PCA classifier and its application in vehicle detection," in Proceedings of the IEEE International Joint Conference on Neural Networks (IJCNN '01), vol. 1, pp. 600604, Washington, DC, USA, July 2001.

[37] A. Khammari, F. Nashashibi, Y. Abramson, and C. Laurgeau, "Vehicle detection combining gradient analysis and AdaBoost classification," in Proceedings of the 8th IEEE International Conference on Intelligent Transportation Systems (ITSC '05), vol. 2005, pp. 66-71, Vienna, Austria, September 2005.

[38] Z. Sun, G. Bebis, and R. Miller, "Object detection using feature subset selection," Pattern Recognition, vol. 37, no. 11, pp. 21652176, 2004.

[39] J. Friedman, T. Hastie, and R. Tibshirani, "Additive logistic regression: a statistical view of boosting," Annals of Statistics, vol. 28, no. 2, pp. 337-407, 2000.

[40] H. Ghorayeb, B. Steux, and C. Laurgeau, "Boosted algorithms for visual object detection on graphics processing units," in Proceedings of the 7th Asian Conference on Computer Vision (ACCV'06), pp. 254-263, Hyderabad, India, January 2006.

[41] M. Fritz, B. Leibe, B. Caputo, and B. Schiele, "Integrating representative and discriminant models for object category detection," in Proceedings of the 10th IEEE International Conference on Computer Vision (ICCV '05), vol. 2, pp. 13631370, Beijing, China, October 2005.

[42] P. Negri, X. Clady, and L. Prevost, "Benchmarking haar and histograms of oriented gradients features applied to vehicle detection," in Proceeding of the 4th International Conference on Informatics in Control, Automation and Robotics (ICINCO '07), pp. 359-364, Angers, France, May 2007.

[43] L. Prevost, L. Oudot, A. Moises, C. Michel-Sendis, and M. Milgram, "Hybrid generative/discriminative classifier for unconstrained character recognition," Pattern Recognition Letters, vol. 26, no. 12, pp. 1840-1848, 2005. 